\title{
Nota Técnica: \\ Estudio de la resistencia eléctrica del sistema traviesa-sujeciones en vías de ferrocarril
}

\section{Technical Note: \\ Study on the electrical resistance of the sleeper-fastening elements system in railway tracks}

\author{
F. J. Barroso ${ }^{(*)}$ P. Guarner(*) y C. López ${ }^{(*)}$
}

Recepción/Received: 11-XI-11

Aceptación/Accepted: 23-I-12

Publicado online/Online publishing: 24-II-12

RESUMEN

En vías de ferrocarril sometidas a condiciones medioambientales desfavorables, la resistencia eléctrica del conjunto formado por una traviesa y el sistema de sujeción del raíl es un parámetro muy importante. Esto se debe a que los raíles actúan como conductores eléctricos en los sistemas de señalización y tracción. Esta resistencia eléctrica, definida como un valor característico del sistema traviesa-elementos de sujeción-agua, se mide con procedimientos normalizados. Sin embargo, estos procedimientos están sujetos a ciertas indefiniciones que provocan una elevada dispersión. En este trabajo se estudia la dependencia de este parámetro en variables tales como la conductividad del agua, la temperatura y la humedad relativa y se establecen estrategias para reducir dicha dispersión a valores mínimos.

Palabras clave: propiedades eléctricas, temperatura, vía de ferrocarril, traviesa, raíl.
SUMMARY

The electrical resistance of the sleeper-fastening elements system in a wet railway track is a very important parameter. This is because the rails are electric conductors in the circuit of signaling and traction systems. This electrical resistance, defined as a characteristic value of the sleeper-fastening elementswater system is a measurand obtained with reference measurement procedures as described in international standards. But it is subject to many kinds of undefinitions that result in a very high dispersion. In this work the dependence of this parameter on variables such as the water conductivity, the temperature and the relative humidity is shown, and several ways to reduce it to minimum values are also established.

Keywords: electrical properties, temperature, railway track, sleeper, rail.

(*) Instituto de Ciencias de la Construcción Eduardo Torroja (IETcC-CSIC) (Madrid, España). 


\section{INTRODUCCIÓN}

En vías ferroviarias convencionales es de vital importancia la resistencia eléctrica de los distintos elementos que componen la vía. Esto es así debido a que determinadas señales utilizan como conductor eléctrico los carriles de la propia vía.

Aunque en condiciones favorables, una traviesa de hormigón y el conjunto de sujeciones que la conectan con el raíl, son aislantes y permiten el buen funcionamiento de dichas señales, en otros casos - lluvia, alta humedad relativa, condensación de humedad, etc.-. los valores de resistencia eléctrica del sistema traviesa-sujeciones pueden ser críticos.

Con el fin de validar tanto a la traviesa como a los distintos elementos del sistema de sujeción, existen normas internacionales que se aplican en distintos ámbitos (por ejemplo, en EEUU se evalúa de acuerdo a la norma "Fastener Electrical Impedance test") (1). Es fundamental, por motivos de seguridad y económicos, que el método de evaluación sea lo más fiable posible.

En las vías de ferrocarril españolas, tanto las convencionales como las de Alta velocidad, es de aplicación la norma UNE-EN 13146-5 (2). En dicho documento se describe detalladamente el procedimiento para obtener un valor de resistencia eléctrica característico del sistema traviesa-sujeciones bajo condiciones atmosféricas desfavorables.

El requisito final, es decir, el valor mínimo de resistencia que debe cumplir el sistema, lo determina en España para las vías de ámbito nacional el organismo administrador de infraestructuras ferroviarias (ADIF) a través de especificaciones técnicas (3).

Sin embargo, debido a la gran cantidad de variables que participan, esta metodología puede presentar una elevada dispersión en los resultados obtenidos. Así, en nuestro laboratorio, después de muchos años realizando este tipo de ensayos hemos encontrado en varias ocasiones que para un mismo sistema su resistencia eléctrica puede alcanzar valores inferiores a la mitad del valor inicial (4) lo que puede provocar que una misma traviesa o un conjunto de sujeciones pueda pasar de cumplir la especificación a no cumplirla.

En la bibliografía existen interesantes estudios que muestran esta variación de la resistencia eléctrica en función de las propiedades físicas del hormigón y de la disposición de las armaduras en su interior (5). En este trabajo se ha estudiado de forma sistemática la influencia sobre la resistencia eléctrica obtenida, de parámetros como la conductividad del agua, su temperatura o la humedad relativa de las sujeciones; se discuten algunos de los factores de mayor importancia en la citada dispersión y finalmente se sugieren métodos para reducirla.

\section{MATERIALES Y MÉTODOS}

\subsection{El ensayo según la norma}

La traviesa con dos cupones de carril y sus elementos de sujeción montados de la misma forma que en la vía, se somete a un rociado con agua, midiéndose durante todo el proceso la resistencia eléctrica entre los dos cupones.

La norma establece condiciones para el rociado (caudal, tipo de rociadores, bastidor móvil, etc.); para la tensión eléctrica $(30 \pm 3$ Vrms a $50 \pm 15 \mathrm{~Hz}$ ) y los equipos de medida (voltímetro y amperímetro con $1 \%$ de precisión, calibrados, etc.); para la temperatura del agua (10-20 $\left.{ }^{\circ} \mathrm{C}\right)$; las condiciones ambientales (15-30 ${ }^{\circ} \mathrm{C}$, a cubierto, sin corrientes de aire pero ventilado, etc.); apoyos de la traviesa (de material aislante de al menos $50 \mathrm{~mm}$ de espesor y bloques de madera); y conductividad del agua a una temperatura de $25^{\circ} \mathrm{C}$ (entre 20 y $80 \mathrm{mS} / \mathrm{m}$ (6)).

El esquema de medida es el que se indica en la Figura 1.

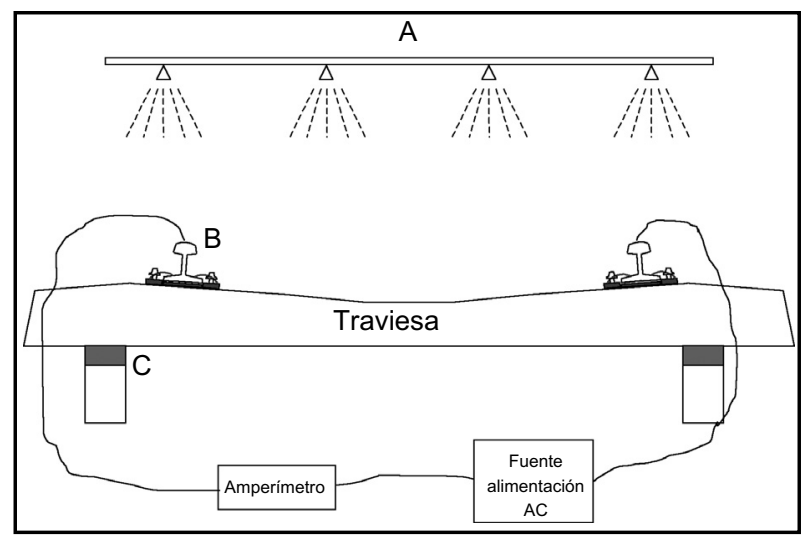

Figura 1. Esquema del circuito de medida de resistencia eléctrica, donde: A) dispositivo de aspersión; B) raíles y elementos de sujeción; C) soportes aislantes.

En cada ensayo se obtiene una gráfica como la que se presenta en la Figura 2. El resultado es el valor mínimo durante el periodo de medida ( 2 minutos de lluvia y al menos 10 minutos posteriormente).

Se deben hacer tres ensayos y calcular la media aritmética. La traviesa debe estar seca superficialmente para ensayar de nuevo y deben pasar al menos 24 horas entre cada ensayo. El resultado obtenido se corrige en función de la conductividad del agua, según la fórmula [1]:

$$
\mathrm{R}=\mathrm{R}_{0} \cdot 0,03 \cdot \gamma
$$

siendo:

$\mathrm{R}$ : resistencia eléctrica resultado del ensayo.

$\mathrm{R}_{0}$ : resistencia eléctrica medida.

$\gamma$ : conductividad del agua. 


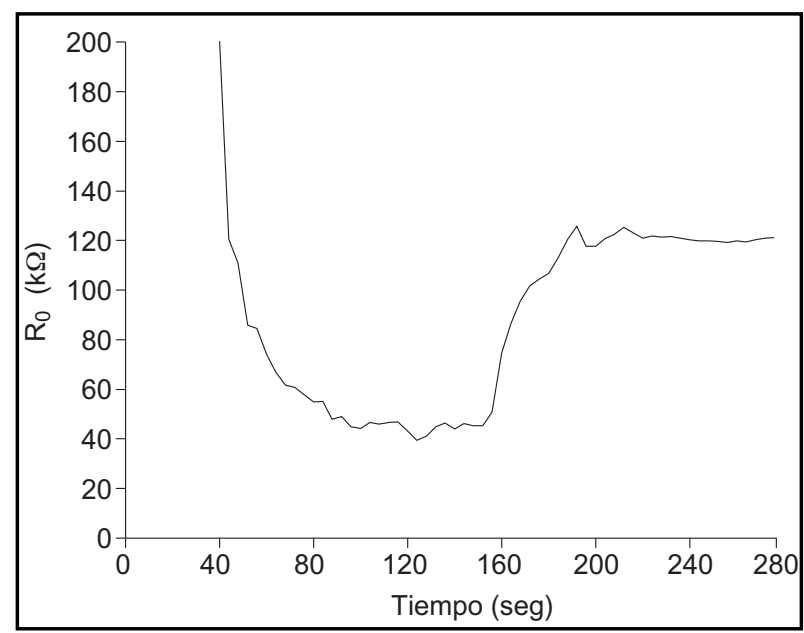

Figura 2. Resistencia eléctrica medida sin corregir por el valor de conductividad.

Así, esta resistencia corregida es el valor correspondiente a una conductividad del agua de $33 \mathrm{mS} / \mathrm{m}$.

\subsection{Equipos empleados}

Para medir la conductividad y la temperatura del agua se utilizó un conductivímetro Lutron CD-4303 calibrado en el intervalo de 20 a $80 \mathrm{mS} / \mathrm{m}$ y de 10 a $25^{\circ} \mathrm{C}$. Este conductivímetro se verificaba regularmente con disoluciones de conductividad conocida $\left(70,6 \mathrm{mS} / \mathrm{m}\right.$ a $25^{\circ} \mathrm{C}$, obtenidas a partir de disoluciones HI 70031 de $141,3 \mathrm{mS} / \mathrm{m}$ a $\left.25^{\circ} \mathrm{C}\right)$.

Para medir el voltaje y la corriente se utilizó un multímetro Agilent 34970A. Este multímetro también dispone de un termopar tipo J para medir la temperatura ambiente.

El sistema de medida se verificó en varias ocasiones sustituyendo la probeta a ensayar por resistencias de $4.7 \mathrm{k} \Omega, 10 \mathrm{k} \Omega$ y $20 \mathrm{k} \Omega$.

La humedad relativa de las placas se midió según el procedimiento indicado en la referencia (7): pesándolas con una balanza de Gram Precision, modelo AH600, antes y después de someterlas a tratamiento térmico en una cámara climática Ineltec CC700.

\subsection{Metodología}

Se llevaron a cabo dos series de ensayos cumpliendo en todo momento las indicaciones de la norma. Se utilizó una traviesa monobloque de hormigón pretensado tipo PR01EA con cupones de carril colocados a la distancia correspondiente al ancho UIC y un mismo conjunto de sujeciones que se mantuvo durante todos los ensayos (placas de asiento PAE-2; placas acodadas: a2/i-60, pa66-gf35 y a2/e-60, pa66-gf35; tornillos de tipo T2 y clips SKL-1).

En la primera serie se evaluaron parámetros como la conductividad, la temperatura del agua, la temperatura ambiente y la resistencia inicial del sistema. Se estudió la variación de resistencia eléctrica al disminuir la conductividad del agua analizando casi todo el intervalo que indica la norma $\left(20-80 \mathrm{mS} / \mathrm{m}\right.$ a $\left.25^{\circ} \mathrm{C}\right)$.

En la segunda serie se trató de mantener constante la humedad relativa del sistema para evitar en lo posible su influencia sobre la resistencia eléctrica obtenida al cambiar la conductividad del agua. Así, después de cada ensayo las sujeciones y los cupones de carril se desmontaban, se limpiaban y se secaban; las placas de asiento y acodadas, se sometían a un tratamiento térmico $\left(100^{\circ} \mathrm{C}\right.$ durante varios días); y los intervalos de tiempo entre cada ensayo se incrementaron hasta llegar a una semana o más.

\section{RESULTADOS}

\subsection{Serie 1}

Los resultados son los que se indican en la Tabla 1.

En la Figura 3 se presentan los valores obtenidos para la resistencia eléctrica corregida $(R)$ y sin corregir $\left(R_{0}\right)$.

En la Tabla 1 se puede apreciar que, aún siguiendo las indicaciones de la norma (Traviesa seca superficialmente y más de $24 \mathrm{H}$ entre ensayo y ensayo), la resistencia inicial del sistema $\left(R_{0 \max }\right)$ varía desde $12 \mathrm{M} \Omega$ hasta $0,3 \mathrm{M} \Omega$. Esto indica que su humedad relativa fue incrementándose con el número de ensayos.

Tabla 1. Resistencia eléctrica medida $\left(R_{0}\right)$, resistencia eléctrica corregida $(R)$, conductividad del agua $(C)$, temperatura del agua ( $\left.T_{\text {agua }}\right)$, temperatura ambiente $\left(T_{a m b}\right)$, resistencia eléctrica antes del rociado $\left(R_{0 \max }\right)$.

\begin{tabular}{|c|c|c|c|c|c|}
\hline $\mathbf{R}_{\mathbf{0}} \mathbf{k} \boldsymbol{\Omega}$ & $\mathbf{R ~} \mathbf{\Omega}$ & $\mathbf{C} \mathbf{~ m S} / \mathbf{m}$ & $\mathbf{T}_{\text {agua }}{ }^{\circ} \mathbf{C}$ & $\mathbf{T}_{\text {amb }}{ }^{\circ} \mathbf{C}$ & $\mathbf{R}_{\mathbf{0 m a x}} \mathbf{M} \boldsymbol{\Omega}$ \\
\hline 20.2 & 41.9 & 69.3 & 17.5 & 17.0 & 12.0 \\
\hline 14.1 & 27.4 & 64.9 & 19.0 & 18.1 & 3.7 \\
\hline 12.0 & 18.6 & 51.7 & 20.2 & 18.8 & 1.5 \\
\hline 13.2 & 16.0 & 40.5 & 21.5 & 22.3 & 0.6 \\
\hline 24.7 & 16.9 & 22.9 & 21.8 & 21.5 & 0.4 \\
\hline 27.3 & 13.3 & 16.2 & 18.7 & 21.8 & 0.3 \\
\hline
\end{tabular}


Así, analizando la Figura 3, si leemos el gráfico de derecha a izquierda, al bajar la conductividad del agua la resistencia eléctrica medida $\left(R_{0}\right)$ debería aumentar. En vez de esto, lo que ocurre es que se mantiene más o menos constante hasta llegar a 40-50 mS/m, donde comienza a subir. Es muy posible que esto se deba al ya citado aumento en la humedad del sistema. Posteriormente sí que se aprecia una subida, posiblemente debida a la saturación de esta humedad y que se refleja en que la resistencia inicial $\left(R_{0 \max }\right)$ permanece en torno a unos cientos de $k \Omega$.

Como resultado, la resistencia corregida $(R)$ presenta una fuerte caída: desde los 42 hasta los $13 \mathrm{k} \Omega$ (entre 70 y $16 \mathrm{mS} / \mathrm{m})$, mientras que la resistencia sin corregir $\left(R_{0}\right)$ presenta una menor variación: de 27 a $12 \mathrm{k} \Omega$ en todo el intervalo.

\subsection{Serie 2}

Los resultados de la serie 2 son los que se indican en la Tabla 2.

En esta serie se puede observar que la resistencia inicial del sistema $\left(R_{0 \max }\right)$ se mantuvo en torno a los $10 \mathrm{M} \Omega$, lo que indica que su grado de humedad fue muy bajo en todos los casos.
Sin embargo, en los primeros ensayos, donde la conductividad del agua estuvo en torno a $80 \mathrm{mS} / \mathrm{m}$, debido al tratamiento térmico de las sujeciones poliméricas (placas de asiento y acodadas), hay una bajada en su humedad relativa desde un $1 \%$ hasta un $0,5 \%$, lo que parece originar un aumento en la resistencia medida. En la figura 4 se presenta este efecto (en la Tabla 2 no se aprecia debido a que los valores indicados son promedio de tres ensayos). En los ensayos posteriores las placas alcanzaron una humedad relativa muy pequeña y que se mantuvo constante durante el resto de la serie, así que no se volvió a apreciar esta subida.

Por otro lado, la diferencia entre la temperatura del agua (del depósito) y la temperatura de la traviesa (ambiente) podría ser otra fuente de error. La explicación es la siguiente: la resistencia eléctrica se corrige según la conductividad medida en el depósito de agua, ahora bien, si la temperatura de la traviesa es diferente, la conductividad del agua al entrar en contacto con ella también será distinta y, por tanto, la corrección que indica la fórmula [1] sería errónea. Este efecto se elimina si las dos temperaturas son iguales. Así, en esta serie se procuró minimizar esta diferencia (Tabla 2).

En la Figura 5 se presentan los valores obtenidos para la resistencia eléctrica antes $\left(R_{0}\right)$ y después $(R)$ de corregir,

Tabla 2. Resistencia eléctrica medida $\left(R_{0}\right)$, resistencia eléctrica corregida $(R)$, conductividad del agua $(C)$, conductividad del agua a $25^{\circ} \mathrm{C}\left(\mathrm{C}_{25}\right)$, humedad relativa de las sujeciones (HR), temperatura del agua ( $\left.T_{\text {agua }}\right)$, temperatura ambiente $\left(T_{\text {amb }}\right)$, resistencia eléctrica antes de la lluvia $\left(R_{0 \max }\right)$.

\begin{tabular}{|c|c|c|c|c|c|c|c|}
\hline $\mathbf{R}_{\mathbf{0}} \mathbf{k} \boldsymbol{\Omega}$ & $\mathbf{R} \mathbf{~} \boldsymbol{\Omega}$ & $\mathbf{C}_{\mathbf{2 5}} \mathbf{~} \mathbf{S} / \mathbf{m}$ & $\mathbf{C} \mathbf{~ m S} / \mathbf{m}$ & $\mathbf{H R} \%$ & $\mathbf{T}_{\text {agua }}{ }^{\circ} \mathbf{C}$ & $\mathbf{T}_{\text {amb }}{ }^{{ }^{\circ} \mathbf{C}}$ & $\mathbf{R}_{\mathbf{0 m a x}} \mathbf{M} \boldsymbol{\Omega}$ \\
\hline 11.6 & 28.4 & 90.0 & 82.1 & 0.78 & 20.6 & 20.3 & 11.1 \\
\hline 17.2 & 34.5 & 78.2 & 66.1 & 0.18 & 17.3 & 18.3 & 12.0 \\
\hline 15.5 & 23.9 & 62.8 & 51.3 & 0.08 & 15.9 & 16.5 & 14.4 \\
\hline 25.1 & 30.6 & 50.1 & 40.5 & 0.06 & 15.4 & 15.5 & 13.2 \\
\hline 39.4 & 33.0 & 34.9 & 27.9 & 0.14 & 15.0 & 15.3 & 13.1 \\
\hline 47.8 & 27.3 & 23.7 & 19.0 & 0.03 & 15.2 & 15.1 & 13.9 \\
\hline
\end{tabular}

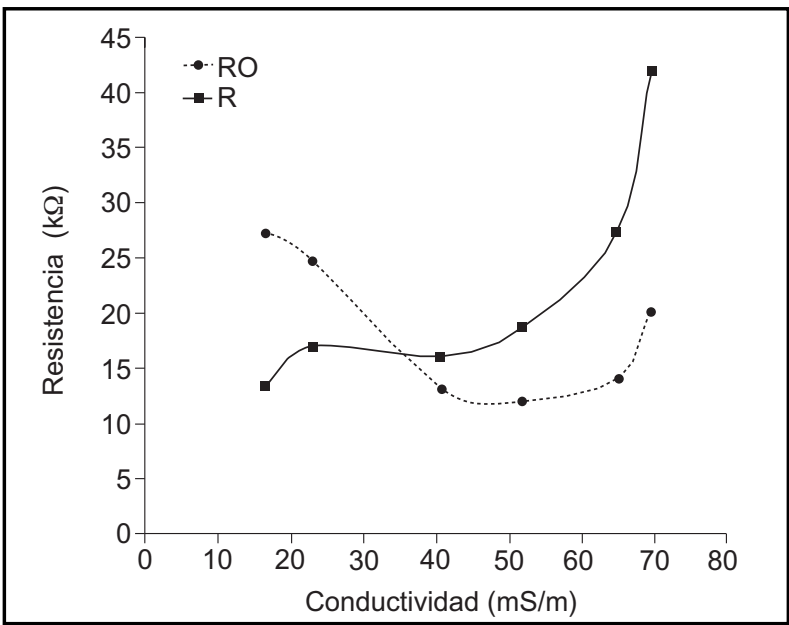

Figura 3. Serie 1: resistencia eléctrica medida $\left(R_{0}\right)$ y corregida (R) en función de la conductividad del agua.

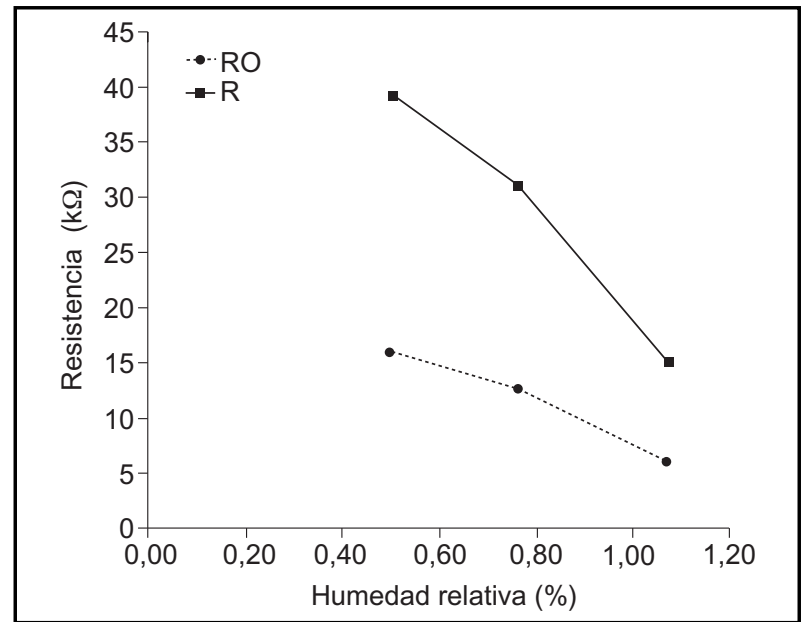

Figura 4. Resistencia eléctrica medida $\left(R_{0}\right)$ y corregida $(R)$ en función de la humedad relativa de las sujeciones en los ensayos iniciales de la serie 2. 


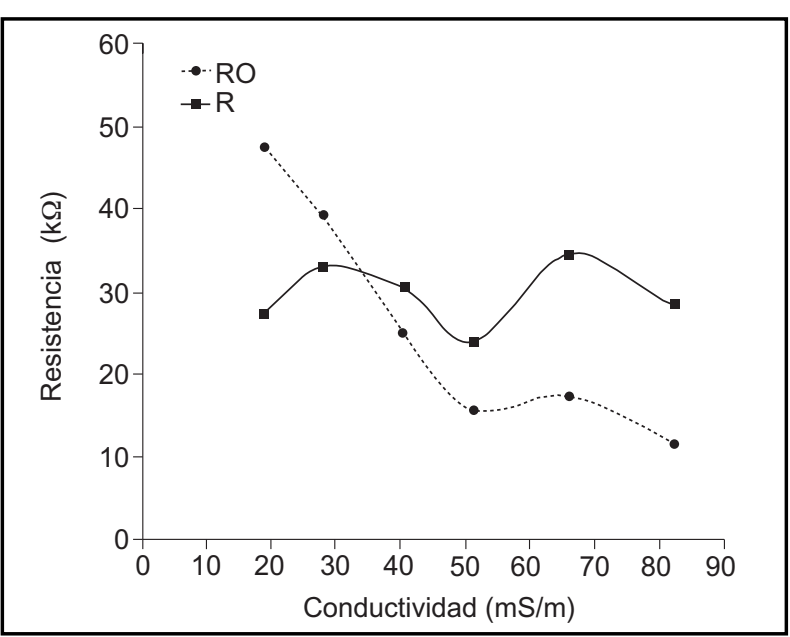

Figura 5. Serie 2: Resistencia eléctrica medida $\left(R_{0}\right)$ y corregida (R) en función de la conductividad del agua.

frente a la conductividad del agua. Se puede observar que en este caso la resistencia medida $\left(R_{0}\right)$ sí que aumenta cuando baja la conductividad del agua y de este modo la resistencia corregida $(R)$ se mantiene constante (con cierta dispersión).

En este caso, $\mathrm{R}_{0}$ sigue presentando un intervalo de variación alto: de $36 \mathrm{k} \Omega$ (de 12 a $48 \mathrm{k} \Omega$ ), mientras que la resistencia corregida $(\mathrm{R})$ presenta un intervalo mucho menor: de $11 \mathrm{k} \Omega$ (de 24 a $35 \mathrm{k} \Omega$ ).

\section{CONCLUSIONES}

Si tenemos en cuenta la definición de "mesurando" según el vocabulario internacional de metrología (8), la filosofía de la norma UNE-EN 13146-5 es obtener el mesurando resistencia eléctrica del sistema traviesa-sujeciones bajo condiciones atmosféricas desfavorables, mediante otros dos mesurandos: a) la resistencia eléctrica real del sistema traviesa-sujeciones-agua y b) la conductividad del agua. Esto simplificando el proceso de medida que hemos estudiado.

Teniendo en cuenta la gran variedad de aguas y de condiciones medioambientales a las que pueden estar sometidas las vías de ferrocarril, el punto de vista de la norma es lógico si se quiere minimizar el efecto de las condiciones de contorno y obtener un valor que pueda ser comparativo entre los distintos sistemas traviesa-sujeciones.
Sin embargo, debido al gran número de variables que participan, la dispersión de los valores obtenidos puede ser muy elevada: en la primera serie de este trabajo, el mismo sistema traviesa-sujeciones presenta un intervalo de variación en la resistencia eléctrica medida de $15 \mathrm{k} \Omega$ (entre 12 y $27 \mathrm{k} \Omega$ ), que aumenta hasta $29 \mathrm{k} \Omega$ (de 13 a $42 \mathrm{k} \Omega$ ) cuando se corrige por el valor de conductividad del agua.

A pesar de todo, si se consideran parámetros como la humedad relativa de la traviesa y de sus sujeciones y se minimizan sus intervalos de variación, esta dispersión disminuye notablemente. Así, en la serie 2 de este trabajo se procuró reducir dicha humedad relativa y el resultado fue que, al variar la conductividad en todo el rango permitido, la resistencia eléctrica medida presentó un intervalo de variación de $36 \mathrm{k} \Omega$, mientras que la resistencia corregida se mantuvo en torno a los $30 \mathrm{k} \Omega$ y su dispersión disminuyó hasta los $11 \mathrm{k} \Omega$.

El problema podría haber sido enfocado desde el punto de vista contrario y quizá más realista, es decir, obligando la saturación de la humedad relativa del sistema. Este sería el caso de la serie 1 de este estudio donde, si analizamos el intervalo de conductividad de 15 a $50 \mathrm{mS} / \mathrm{m}$, el valor de R estaría en torno a $17 \mathrm{k} \Omega$ con una dispersión de $5 \mathrm{k} \Omega$.

Por otro lado, es fundamental controlar minuciosamente la temperatura del ensayo y homogeneizar las temperaturas del agua y del ambiente. Esto se debe a la fuerte dependencia de la conductividad del agua con esta variable. Véase por ejemplo, en la tabla 2, la diferencia entre la conductividad a la temperatura del depósito y a $25^{\circ} \mathrm{C}$. Teniendo en cuenta esta diferencia, pueden aparecer errores significativos en la medida de R. Por ejemplo, en la tabla 2 podemos observar que para la misma agua, a $15,0^{\circ} \mathrm{C}$ la conductividad es de $27,9 \mathrm{mS} / \mathrm{m}$ obteniéndose una resistencia de $33 \mathrm{k} \Omega$, mientras que a $25^{\circ} \mathrm{C}$ la $\mathrm{C}_{25}$ sería $34,9 \mathrm{mS} / \mathrm{m}$, y el resultado sería de $41 \mathrm{k} \Omega$.

\section{AGRADECIMIENTOS}

Queremos agradecer a nuestros compañeros Gabriel Rentero y Rafael Soldado su importante colaboración durante este trabajo.

\section{BIBLIOGRAFÍA}

(1) "Fastener Electrical impedance test". Manual for railway engineering, vol. 1, Track. Ch 30 Ties. 2.8. Test 7. AREMA (2010).

(2) "Aplicaciones ferroviarias. Vía. Métodos de ensayo de los sistemas de fijación. Parte 5: Determinación de la resistencia eléctrica". UNE-EN 13146-5: 2003. 
(3) "Traviesas monobloque de hormigón pretensado". ADIF. VIA. Especificaciones técnicas. ET 03.360.571.8. 4a edición: enero de 2009. (4) López, C.; Barroso, F. J. y Zamora, A.: "Ensayos sobre modelos de traviesas de desvío de hormigón pretensado". IETCC. Informe técnico 19517 (2009).

(5) Carrascal, I. A.; Casado, J. A.; Polanco, J. A. y Gutiérrez-Solana, F.: "Efecto de las características físico-geométricas de las traviesas de hormigón monobloque en el comportamiento eléctrico de la superestructura de vía de ferrocarril para AVE". Materiales Compuestos 05. AEMAC. Valencia (2005), pp. 667-674.

(6) "Calidad del agua. Determinación de la conductividad eléctrica". EN 27888 (ISO 7888: 1985).

(7) "Plastics. Determination of Water Absorption". ISO 62: 2008.

(8) International vocabulary of metrology. Basic and general concepts and associated terms. (VIM). JCGM 200: 2008. 